\title{
Borboletas (Lepidoptera: Papilionoidea e Hesperioidea) ocorrentes em diferentes ambientes na Floresta Ombrófila Mista e nos Campos de Cima da Serra do Rio Grande do Sul, Brasil
}

\author{
Cristiano Agra Iserhard ${ }^{1,3}$, Marina Todeschini de Quadros ${ }^{1}$, \\ Helena Piccoli Romanowski ${ }^{1}$ \& Milton de Souza Mendonça Jr. ${ }^{2}$ \\ IPrograma de Pós-graduação em Biologia Animal, Departamento de Zoologia, \\ Universidade Federal do Rio Grande do Sul - UFRGS, \\ Av. Bento Gonçalves, 9500, Prédio 43435, Laboratório 218, CEP 91501-970 Porto Alegre, RS, Brasil \\ ${ }^{2}$ Departamento de Ecologia, Universidade Federal do Rio Grande do Sul-UFRGS, \\ Av. Bento Gonçalves, 9500, Prédio 43422 CEP 91501-970, Porto Alegre, RS, Brasil \\ ${ }^{3}$ Autor para correspondência: Cristiano Agra Iserhard, e-mail: cristianoagra@yahoo.com.br
}

ISERHARD, C.A., QUADROS, M.T., ROMANOWSKI, H.P. \& MENDONÇA Jr. M.S. Occurrence of butterflies (Lepidoptera: Papilionoidea and Hesperioidea) in different habitats at the Araucaria Moist Forest and the Grasslands in the Basaltic Highlands in Southern Brazil. Biota Neotrop. 10(1): http://www.biotaneotropica. org.br/v10n1/en/abstract?inventory+bn02910012010.

\begin{abstract}
Aiming to contribute to the knowledge of Araucaria Moist Forest butterflies in Rio Grande do Sul, a butterfly species list of the São Francisco National Forest and surroundings was compiled. Field expeditions were carried out bimonthly between March 2006 to May 2008 in six types of environments: (i) Araucaria angustifolia native forest, (ii) native forest mixed with Araucaria angustifolia plantation, (iii) Araucaria angustifolia plantation in open forest, (iv) Araucaria angustifolia plantation in closed forest, (v) Pinus plantation and (vi) grasslands in the basaltic highlands. After 674 net-hours of sampling effort 277 species and subspecies distributed in 9661 individuals, belonging to six families of butterflies were registered. One hundred thirty-nine species are new records for this region of Atlantic Forest and 13 species are new registers for Rio Grande do Sul State. Six species are rare and/or healthy environment indicators.
\end{abstract}

Keywords: altitudinal grasslands, Araucaria Forest, butterfly conservation, National Forest, silviculture, species richness.

ISERHARD, C.A., QUADROS, M.T., ROMANOWSKI, H.P. \& MENDONÇA Jr. M.S. Borboletas (Lepidoptera: Papilionoidea e Hesperioidea) ocorrentes em diferentes ambientes na Floresta Ombrófila Mista e nos Campos de Cima da Serra do Rio Grande do Sul, Brasil. Biota Neotrop. 10(1): http://www.biotaneotropica. org.br/v10n1/pt/abstract?inventory+bn02910012010.

Resumo: Com o intuito de contribuir para o conhecimento das borboletas da Floresta Ombrófila Mista e Campos de Cima da Serra do Rio Grande do Sul foi elaborada uma listagem de espécies da assembléia de borboletas da Floresta Nacional de São Francisco de Paula e entorno. Foram realizadas saídas bimestrais de março de 2006 a maio de 2008 sendo selecionados seis ambientes: (i) mata nativa de Araucaria angustifolia, (ii) mata nativa mesclada com reflorestamento de Araucaria angustifolia, (iii) reflorestamento de Araucaria angustifolia de mata aberta, (iv) reflorestamento de Araucaria angustifolia de mata fechada, (v) reflorestamento de Pinus e (vi) campo de altitude nativo. Após 674 horas-rede de amostragem foram registradas 277 espécies e subespécies de borboletas, distribuídas em 9661 indivíduos, pertencentes a seis famílias, sendo destas 139 novos registros para esta região dos Campos de Cima da Serra, 13 novas ocorrências para o estado e seis espécies raras ou indicadoras de ambiente preservado.

Palavras-chave: campos de altitude, conservação de borboletas, Floresta com Araucária, Floresta Nacional, riqueza de espécies, silvicultura. 


\section{Introdução}

Gerar informações sobre a biodiversidade é fundamental para manter a ligação entre o conhecimento científico e a tomada de decisões em prol da conservação (Backes \& Irgang 2004). Inventários de fauna em curtos períodos de tempo resultam geralmente em listagens de espécies e permitem, posteriormente, o monitoramento desta fauna ao longo do tempo, avaliando possíveis mudanças.

As borboletas, além de ser um dos grupos de invertebrados mais estudados (Boggs et al. 2003, Ockinger et al. 2006) apresentam características que as tornam excelentes ferramentas no monitoramento da qualidade ambiental; são bastante diversificadas, relativamente fáceis de amostrar e identificar, presentes ao longo do ano e, principalmente, respondem com rapidez a distúrbios ou alterações no ambiente (Brown 1996, New 1997, Ockinger et al. 2006).

O bioma Mata Atlântica ocupa $15 \%$ do território brasileiro e apresenta uma variedade de formações, dentre elas, a Floresta Ombrófila Mista e os Campos de Cima da Serra ou Campos de Altitude. A Floresta Ombrófila Mista (Mata com Araucária) é uma das mais importantes formações florestais do sul do Brasil. No Rio Grande do Sul as áreas de floresta primária são poucas e, em geral, alteradas. Foram substituídas por culturas cíclicas e permanentes, pastagens, reflorestamentos (com Pinus sp. e Eucalyptus sp.) e vegetação secundária (Leite 2002); atualmente vem sendo destruída por práticas não sustentáveis como a expansão da indústria, do turismo e da urbanização desordenada (Bond-Buckup 2008).

Os Campos de Cima da Serra, igualmente ameaçados, vêm sendo substituídos pela agricultura, pecuária e intensa silvicultura. São áreas de alto endemismo, devido à preferência de determinados organismos por habitats especializados, aliados aos mecanismos de isolamento geográfico nestas regiões (Safford 1999, 2007, Behling 2002). Pouco se sabe a respeito da sua biodiversidade no Rio Grande do Sul, sendo esses dados prioritários para o estabelecimento de subsídios e programas de manejo e conservação (Bond-Buckup 2008).

Na região dos Campos de Cima da Serra, a espécie mais utilizada na silvicultura é o Pinus elliotii Engelm. As extensões de monoculturas arbóreas causam o esgotamento do solo, a alteração no escoamento e infiltração da água e sua evapotranspiração, além de diminuir a biodiversidade impedindo a recuperação e diversificação da fauna pela homogeneidade de suas florestas. Por serem cultivadas em áreas de campos e matas nativas, sem qualquer levantamento prévio da fauna local, destroem um bioma único e já bastante antropizado e descaracterizado (Bond-Buckup 2008).

Romanowski et al. (2009) elaboraram uma lista de espécies de borboletas através de informações da literatura, registros históricos e amostragens de campo de diferentes regiões de Floresta Ombrófila Mista e Campos de Cima da Serra e chegaram a um total de 162 espécies. Recentemente uma compilação de dados coletados em campo gerou a publicação de um livro com informações a respeito da flora e fauna (incluindo borboletas) dos Campos de Cima da Serra (Bond-Buckup 2008). A fauna de borboletas desta região do Rio Grande do Sul foi estudada também no Centro de Pesquisas e Conservação da Natureza Pró-Mata, São Francisco de Paula, por Teston \& Corseuil (1999, 2000a, 2002a) e Corseuil et al. (2004). Porém, a família Hesperiidae não foi contemplada nestes estudos, além de não haver menção ao esforço amostral, nem caracterização dos locais de amostragem. Segundo Iserhard \& Romanowski (2004) estes são problemas comuns evidenciados em alguns trabalhos faunísticos de borboletas no Rio Grande do Sul.

Com o intuito de dar continuidade aos estudos relativos a esta região, este trabalho tem como objetivos (i) ampliar a área efetivamente estudada dos Campos de Cima da Serra e da Floresta Ombrófila Mista do Rio Grande do Sul, através da elaboração de uma lista de espécies de borboletas da Floresta Nacional de São Francisco de Paula e entorno; (ii) contribuir para o conhecimento desta fauna a partir de informações de registros não publicados para a região e para o Estado, bem como verificar a presença de espécies de borboletas indicadoras de ambientes preservados.

\section{Material e Métodos}

\section{1. Área de estudo}

A Floresta Nacional de São Francisco de Paula (FLONA) (292 $24^{\prime}$ S e $50^{\circ} 22^{\prime}$ W) é uma Unidade de Conservação localizada no nordeste do Rio Grande do Sul, na microrregião dos Campos de Cima da Serra, município de São Francisco de Paula (Fernandes \& Backes 1998), fazendo parte do Planalto Sul-riograndense. Compreende uma área de 1606,60 ha, a $900 \mathrm{~m}$ acima do nível do mar. O clima é do tipo temperado $(\mathrm{Cfb})$ com temperatura média anual de $14,5^{\circ} \mathrm{C}$ (Backes 1999) e altos níveis de pluviosidade em todos os meses, com média de $2252 \mathrm{~mm}$ por ano.

\section{Amostragem}

Foram selecionados seis ambientes na FLONA: mata nativa de Araucaria angustifolia (Bertol.) Kuntze (MN), mata nativa mesclada com reflorestamento de Araucaria angustifolia (NR), reflorestamento de Araucaria angustifolia de mata aberta (RA) e mata fechada (RF) e reflorestamento de Pinus (RP). O ambiente de campo de altitude nativo $(\mathrm{CN})$ foi selecionado no entorno da FLONA em uma propriedade particular vizinha. Todos os ambientes escolhidos tiveram o intuito de englobar e representar as diferentes fisionomias encontradas na área de estudo e na região como um todo. A mata nativa consiste em florestas fechadas no dossel, com baixa incidência solar e poucos recursos alimentares (flores) para adultos de borboletas. As transecções de reflorestamento de araucária eram percorridas ao longo de vias de acesso abertas, com luminosidade variável, e muitos recursos alimentares para as borboletas nas bordas das mesmas. Os Pinus se caracterizam por serem matas homogêneas com ausência de sub-bosque, baixa incidência solar e domínio amplo de Pinus elliotti. As trilhas de campo eram características dos ambientes nativos desta região, sofrendo apenas a influência do pastejo extensivo.

Foram realizadas amostragens bimestrais de março de 2006 a maio de 2008. Cada ambiente era representado por duas transeções, sendo que quatro ambientes (MN, NR, RA e RF) foram amostrados ao longo de dois anos (março 2006 a fevereiro de 2008) e dois ambientes (RP e CN) foram amostrados ao longo de um ano (julho 2007 a maio 2008). As transeções eram percorridas com esforço amostral padronizado em 2 horas/rede, sempre entre 10:00 e 16:30 horas. Borboletas visualizadas eram registradas e, se necessário, coletadas com auxílio de redes entomológicas. Maiores detalhes sobre o protocolo de amostragem são descritos em Paz et al. (2008). Os espécimes coletados estão depositados na coleção de referência de Lepidoptera do Departamento de Zoologia da Universidade Federal do Rio Grande do Sul.

\section{Análise dos dados}

Para comparação da listagem de espécies gerada neste estudo, foram consultados os trabalhos de Weymer (1894), Mabilde (1896), Biezanko (1958, 1959a, b, 1960a, b, c, d, e, 1963), Biezanko \& Mielke (1973), Biezanko et al. (1978), Mielke (1980a, b), Teston \& Corseuil (1998, 1999, 2000a, b, 2001, 2002a, b, 2008a, b, c), Di Mare et al. (2003), Kruger \& Silva (2003), Corseuil et al. (2004), Iserhard \& Romanowski (2004), Quadros et al. (2004), Francini \& Penz (2006), Marchiori \& Romanowski (2006), Teston et al. (2006), Dessuy \& Morais (2007), Giovernadi et al. (2008), Paz 
et al. (2008), Romanowski et al. (2009). A classificação utilizada para a elaboração da lista de espécies seguiu Lamas (2004, 2008) e Mielke (2005). A determinação de espécies raras ou indicadoras de ambientes preservados seguiu Brown \& Freitas (2000a) e Grazia et al. (2008) e a indicação de sistematas. Para comparação com outros trabalhos, em determinadas análises as famílias Lycaenidae e Riodinidae foram agrupadas. Foram calculados, através do Software EstimateS 8.0 (Colwell 2007), os estimadores analíticos de riqueza de espécies Jackknife 2 e Michaelis-Menten para avaliar o quanto da assembléia de borboletas foi contemplada na área de estudo

\section{Resultados e Discussão}

Com 674 horas-rede de amostragem, foram registrados 9661 indivíduos, distribuídos em 277 espécies de borboletas, pertencentes a seis famílias e 22 subfamílias para a Floresta Nacional de São Francisco de Paula e para a região dos Campos de Cima da Serra (Tabela 1). De acordo com os valores de riqueza máximo (Jackknife 2) e mínimo (Michaelis-Menten) dos estimadores analíticos calculados, entre $68 \%$ e $93 \%$ das espécies de borboletas da FLONA e entorno foram amostradas. Teston \& Corseuil (1999, 2000a, 2002a) e Corseuil et al. (2004) registram 103 espécies de borboletas para o Centro de Pesquisas e Conservação da Natureza Pró-Mata (CPCN), distante aproximadamente $20 \mathrm{~km}$ da área do presente estudo. Como mencionado anteriormente, estes autores não incluíram Hesperiidae em suas análises, tampouco mencionam esforço amostral, mesmo assim, a diferença proporcional às demais famílias na riqueza de espécies é considerável.

A família com maior representatividade foi Nymphalidae, seguida de Hesperiidae, Lycaenidae + Riodinidae, Pieridae e Papilionidae (Tabela 1). A frequiência relativa da riqueza de espécies segue um padrão semelhante ao registrado por Iserhard \& Romanowski
(2004), em inventário realizado ao longo de um ano em uma região de Floresta Ombrófila Densa no Vale do rio Maquiné, Rio Grande do Sul (Tabela 2). Porém, tais resultados diferem do registrado para Morais et al. (2007), para a região austral da América do Sul (Argentina, Uruguai e Rio Grande do Sul), onde há uma inversão de representatividade, com Hesperiidae superando Nymphalidae e para o Brasil, onde as três famílias mais ricas em espécies são respectivamente Lycaenidae, Hesperiidae e Nymphalidae (Brown \& Freitas 1999) (Tabela 2).

Chama a atenção a acentuada diferença entre as proporções de riqueza de espécies de Nymphalidae e Hesperiidae para os Campos de Cima da Serra e para o Vale do rio Maquiné (Iserhard \& Romanowski 2004), a menor proporção de Lycaenidae + Riodinidae e um aumento substancial na proporção de Pieridae quando comparadas aos demais trabalhos (Tabela 2). Ressalta-se que a Floresta Ombrófila Mista e os Campos de Altitude possuem características peculiares de vegetação e, principalmente, clima, com sazonalidade marcada e grande oscilação de temperatura. Segundo Brown \& Freitas (2000b) em amplo estudo sobre borboletas da Mata Atlântica, há uma tendência para o aumento na riqueza de espécies de Nymphalidae, Pieridae e Papilionidae em regiões de temperaturas médias mais baixas e maior amplitude térmica.

Teston \& Corseuil (1999, 2000a, 2002a) e Corseuil et al. (2004) registram 64 espécies de Nymphalidae, 17 espécies de Pieridae, seis espécies de Papilionidae e 16 espécies de Lycaenidae para o CPCN. Destas, seis espécies de ninfalídeos, duas de pierídeos e cinco de licenídeos não foram registradas neste estudo. Por outro lado, aqui, 139 registros são novos para os Campos de Cima da Serra e Floresta Ombrófila Mista do Rio Grande do Sul, sendo 34 Nymphalidae, nove Pieridae, quatro Papilionidae, 17 Lycaenidae, 14 Riodinidae e 61 Hesperiidae (Tabela 1) e 13 espécies são novas ocorrências para o Estado (Tabela 3).

Tabela 1. Lista de espécies de borboletas da Floresta Ombrófila Mista e Campos de Cima da Serra.

Table 1. Butterfly species list in the Araucária Moist Forest and Grasslands in the Basaltic Highlands.

\begin{tabular}{|c|c|c|c|c|c|c|}
\hline \multirow[t]{2}{*}{ Famílias/Subfamílias/Espécies } & \multicolumn{6}{|c|}{ Ambientes } \\
\hline & MN & NR & RA & $\mathbf{R F}$ & $\mathbf{R P}$ & $\mathbf{C N}$ \\
\hline \multicolumn{7}{|l|}{ NYMPHALIDAE (S = 108) } \\
\hline \multicolumn{7}{|l|}{ Danainae $(\mathrm{S}=2)$} \\
\hline Danaus erippus (Cramer, 1775) & - & - & $X$ & - & $\mathrm{X}$ & $\mathrm{X}$ \\
\hline Lycorea ilione (Cramer, 1775) & $\mathrm{X}$ & - & - & $\mathrm{X}$ & $\mathrm{X}$ & - \\
\hline \multicolumn{7}{|l|}{ Ithomiinae $(\mathrm{S}=13)$} \\
\hline *Aeria olena Weyer, 1875 & - & - & $\mathrm{X}$ & - & - & - \\
\hline Dircenna dero (Hübner, 1823) & $\mathrm{X}$ & $\mathrm{X}$ & $\mathrm{X}$ & $\mathrm{X}$ & - & - \\
\hline Episcada carcinia Schaus, 1902 & $\mathrm{X}$ & - & - & $X$ & - & - \\
\hline Episcada hymenaea hymenaea (Prittwitz, 1865) & $\mathrm{X}$ & $\mathrm{X}$ & $\mathrm{X}$ & $\mathrm{X}$ & - & - \\
\hline Episcada philoclea (Hewitson, 1855) & $\mathrm{X}$ & - & - & $\mathrm{X}$ & - & - \\
\hline Epityches eupompe (Geyer, 1832) & $\mathrm{X}$ & $\mathrm{X}$ & $\mathrm{X}$ & $\mathrm{X}$ & $\mathrm{X}$ & $\mathrm{X}$ \\
\hline *Hyalenna pascua(Schaus, 1902) & - & - & - & $\mathrm{X}$ & - & - \\
\hline *Ithomia drymo Hübner, 1816 & - & - & - & $\mathrm{X}$ & - & - \\
\hline Mechanitis lysimnia lysimnia (Fabricius, 1793) & $\mathrm{X}$ & $\mathrm{X}$ & $\mathrm{X}$ & $\mathrm{X}$ & $\mathrm{X}$ & $\mathrm{X}$ \\
\hline Methona themisto (Hübner, 1818) & $\mathrm{X}$ & $\mathrm{X}$ & $\mathrm{X}$ & - & - & - \\
\hline *Placidina euryanassa C. Felder \& R. Felder, 1860 & - & - & $\mathrm{X}$ & $\mathrm{X}$ & - & - \\
\hline Pseudoscada erruca (Hewitson, 1855) & $\mathrm{X}$ & $\mathrm{X}$ & $\mathrm{X}$ & $\mathrm{X}$ & - & $\mathrm{X}$ \\
\hline Pteronymia carlia (Schaus, 1902) & $\mathrm{X}$ & $\mathrm{X}$ & $\mathrm{X}$ & $\mathrm{X}$ & - & - \\
\hline
\end{tabular}

Lista de espécies de borboletas registradas de março de 2006 a maio de 2008 na Floresta Nacional de São Francisco de Paula e entorno. MN, mata nativa; NR, mata nativa mesclada com reflorestamento de Araucária; RA, reflorestamento de Araucária de mata aberta; RF, Reflorestamento de Araucária de mata fechada; RP, reflorestamento de Pinus; CN, Campo nativo; S, riqueza de espécies. * novos registros para a região dos Campos de Cima da Serra. 
Tabela 1. Continuação...

\begin{tabular}{|c|c|c|c|c|c|c|}
\hline \multirow[t]{2}{*}{ Famílias/Subfamílias/Espécies } & \multicolumn{6}{|c|}{ Ambientes } \\
\hline & MN & NR & RA & RF & $\mathbf{R P}$ & $\mathbf{C N}$ \\
\hline \multicolumn{7}{|l|}{ Morphinae $(\mathrm{S}=8)$} \\
\hline Blepolenis bassus (Felder \& Felder, 1867) & - & - & $\mathrm{X}$ & $\mathrm{X}$ & - & - \\
\hline Caligo martia (Godart, 1824) & $\mathrm{X}$ & - & $\mathrm{X}$ & - & - & - \\
\hline *Catoblepia amphirhoe (Hübner, 1825) & - & - & - & $\mathrm{X}$ & - & - \\
\hline Eryphanis reevesii (Doubleday, 1849) & - & - & $\mathrm{X}$ & $\mathrm{X}$ & - & - \\
\hline *Morpho aega (Hübner, 1822) & - & $\mathrm{X}$ & - & $\mathrm{X}$ & $\mathrm{X}$ & - \\
\hline Morpho epistrophus catenaria (Perry, 1811) & $\mathrm{X}$ & $\mathrm{X}$ & $\mathrm{X}$ & $\mathrm{X}$ & $\mathrm{X}$ & $\mathrm{X}$ \\
\hline Morpho portis thamyris (Felder \& Felder, 1867) & $\mathrm{X}$ & $\mathrm{X}$ & $\mathrm{X}$ & $\mathrm{X}$ & $\mathrm{X}$ & $\mathrm{X}$ \\
\hline Opoptera fruhstorferi (Röber, 1896) & - & - & - & $\mathrm{X}$ & - & - \\
\hline \multicolumn{7}{|l|}{ Satyrinae $(S=21)$} \\
\hline *Capronnieria galesus (Godart, 1824) & - & - & $\mathrm{X}$ & - & $\mathrm{X}$ & $\mathrm{X}$ \\
\hline Eteona tisiphone (Boisduval, 1836) & $\mathrm{X}$ & $\mathrm{X}$ & - & - & - & - \\
\hline *Euptychoides castrensis (Schaus, 1902) & $\mathrm{X}$ & $\mathrm{X}$ & $\mathrm{X}$ & $\mathrm{X}$ & $\mathrm{X}$ & $\mathrm{X}$ \\
\hline Forsterinaria necys (Godart, 1824) & $\mathrm{X}$ & $\mathrm{X}$ & $\mathrm{X}$ & $\mathrm{X}$ & $\mathrm{X}$ & - \\
\hline Guaianaza pronophila (Butler, 1867) & $\mathrm{X}$ & $\mathrm{X}$ & $\mathrm{X}$ & $\mathrm{X}$ & $\mathrm{X}$ & $\mathrm{X}$ \\
\hline Forsterinaria quantius (Godart, 1824) & - & $X$ & $X$ & $\mathrm{X}$ & - & - \\
\hline Hermeuptychia hermes (Fabricius, 1775) & $\mathrm{X}$ & $\mathrm{X}$ & $\mathrm{X}$ & $\mathrm{X}$ & $\mathrm{X}$ & $\mathrm{X}$ \\
\hline Moneuptychia griseldis (Weymer, 1911) & $\mathrm{X}$ & $\mathrm{X}$ & $\mathrm{X}$ & $\mathrm{X}$ & $\mathrm{X}$ & - \\
\hline Moneuptychia paeon (Godart, 1824) & $\mathrm{X}$ & $\mathrm{X}$ & $\mathrm{X}$ & $\mathrm{X}$ & $\mathrm{X}$ & $\mathrm{X}$ \\
\hline *Moneuptychia soter (Butler, 1877) & - & $\mathrm{X}$ & $\mathrm{X}$ & $\mathrm{X}$ & $\mathrm{X}$ & - \\
\hline Pampasatyrus ocelloides (Schaus, 1902) & - & - & - & - & - & $\mathrm{X}$ \\
\hline Pampasatyrus periphas (Godart, 1824) & - & - & - & - & $\mathrm{X}$ & $\mathrm{X}$ \\
\hline Pampasatyrus quies (Berg, 1877) & - & - & - & - & - & $\mathrm{X}$ \\
\hline Pampasatyrus reticulata (Weymer, 1907) & - & - & - & - & - & $\mathrm{X}$ \\
\hline Paryphthimoides phronius (Godart, 1824) & $\mathrm{X}$ & $\mathrm{X}$ & $\mathrm{X}$ & $\mathrm{X}$ & $\mathrm{X}$ & $\mathrm{X}$ \\
\hline *Paryphthimoides sp. & $\mathrm{X}$ & - & $\mathrm{X}$ & $\mathrm{X}$ & $\mathrm{X}$ & - \\
\hline Praepedaliodes phanias (Hewitson, 1862) & - & - & $\mathrm{X}$ & $\mathrm{X}$ & - & $X$ \\
\hline *Satyrinae sp. & - & - & - & - & - & $\mathrm{X}$ \\
\hline Taygetis ypthima Hübner, 1821 & $\mathrm{X}$ & - & - & $X$ & - & - \\
\hline Yphthimoides ochracea (Butler, 1867) & - & - & $X$ & $\mathrm{X}$ & $X$ & $X$ \\
\hline *Yphthimoides viviana (Romieux, 1927) & - & - & - & - & - & $X$ \\
\hline \multicolumn{7}{|l|}{ Charaxinae $(S=6)$} \\
\hline *Archaeoprepona amphimachus (Fabricius, 1775) & - & - & - & - & $\mathrm{X}$ & - \\
\hline Archaeoprepona chalciope (Hübner, 1823) & $\mathrm{X}$ & - & $\mathrm{X}$ & - & - & - \\
\hline *Archaeoprepona demophon (Linnaeus, 1758) & $\mathrm{X}$ & - & - & - & - & - \\
\hline Memphis hirta (Weymer, 1907) & $\mathrm{X}$ & $\mathrm{X}$ & $\mathrm{X}$ & - & $\mathrm{X}$ & $\mathrm{X}$ \\
\hline *Memphis moruus stheno (Prittwitz, 1865) & - & $X$ & - & $\mathrm{X}$ & - & $\mathrm{X}$ \\
\hline Zaretis itys itylus (Westwood, 1850) & - & - & $\mathrm{X}$ & $\mathrm{X}$ & - & - \\
\hline \multicolumn{7}{|l|}{ Biblidinae $(\mathrm{S}=10)$} \\
\hline Biblis hyperia (Cramer, 1779) & - & - & $\mathrm{X}$ & $\mathrm{X}$ & - & - \\
\hline Catonephele sabrina (Hewitson, 1852) & - & $\mathrm{X}$ & - & - & - & - \\
\hline Diaethria clymena meridionalis (H.W.Bates, 1864) & - & - & - & - & - & $X$ \\
\hline Dynamine myrrhina (Doubleday, 1849) & $\mathrm{X}$ & $X$ & $X$ & $\mathrm{X}$ & - & - \\
\hline Epiphile hubneri Hewitson, 1861 & $\mathrm{X}$ & $\mathrm{X}$ & $\mathrm{X}$ & $\mathrm{X}$ & - & $X$ \\
\hline *Epiphile orea (Hübner, 1823) & $\mathrm{X}$ & $\mathrm{X}$ & - & - & - & - \\
\hline Eunica eburnea Fruhstorfer, 1907 & $\mathrm{X}$ & $\mathrm{X}$ & $\mathrm{X}$ & - & - & $\mathrm{X}$ \\
\hline *Haematera pyrame (Hübner, 1819) & - & - & - & $\mathrm{X}$ & - & - \\
\hline *Hamadryas amphinome amphinome (Linnaeus, 1767) & - & - & $\mathrm{X}$ & $\mathrm{X}$ & - & - \\
\hline
\end{tabular}

Lista de espécies de borboletas registradas de março de 2006 a maio de 2008 na Floresta Nacional de São Francisco de Paula e entorno. MN, mata nativa; NR, mata nativa mesclada com reflorestamento de Araucária; RA, reflorestamento de Araucária de mata aberta; RF, Reflorestamento de Araucária de mata fechada; RP, reflorestamento de Pinus; CN, Campo nativo; S, riqueza de espécies. * novos registros para a região dos Campos de Cima da Serra. 
Tabela 1. Continuação...

\begin{tabular}{|c|c|c|c|c|c|c|}
\hline \multirow[t]{2}{*}{ Famílias/Subfamílias/Espécies } & \multicolumn{6}{|c|}{ Ambientes } \\
\hline & $\mathbf{M N}$ & NR & $\mathbf{R A}$ & RF & $\mathbf{R P}$ & $\mathbf{C N}$ \\
\hline Hamadryas epinome (Felder \& Felder, 1867) & - & $\mathrm{X}$ & $\mathrm{X}$ & $\mathrm{X}$ & $\mathrm{X}$ & - \\
\hline \multicolumn{7}{|l|}{ Apaturinae $(S=3)$} \\
\hline Doxocopa kallina (Staundiger, 1886) & - & - & $X$ & $\mathrm{X}$ & - & - \\
\hline Doxocopa laurentia (Godart, 1824) & $\mathrm{X}$ & $\mathrm{X}$ & $\mathrm{X}$ & $\mathrm{X}$ & $\mathrm{X}$ & $\mathrm{X}$ \\
\hline Doxocopa zunilda (Godart, 1824) & - & - & $X$ & - & - & - \\
\hline \multicolumn{7}{|l|}{ Nymphalinae $(S=17)$} \\
\hline Anartia amathea roeselia (Eschsholtz, 1821) & $\mathrm{X}$ & $\mathrm{X}$ & $\mathrm{X}$ & $\mathrm{X}$ & $\mathrm{X}$ & $\mathrm{X}$ \\
\hline Anartia jatrophae (Linnaeus, 1763) & - & - & $\mathrm{X}$ & - & - & - \\
\hline Eresia lansdorfi (Godart, 1819) & $\mathrm{X}$ & - & $\mathrm{X}$ & $\mathrm{X}$ & - & $\mathrm{X}$ \\
\hline Hypanartia bella (Fabricius, 1793) & $\mathrm{X}$ & $\mathrm{X}$ & $\mathrm{X}$ & $\mathrm{X}$ & $\mathrm{X}$ & $\mathrm{X}$ \\
\hline Hypanartia lethe (Fabricius, 1793) & $\mathrm{X}$ & - & $\mathrm{X}$ & $\mathrm{X}$ & - & - \\
\hline Junonia evarete (Cramer, 1779) & - & - & $\mathrm{X}$ & - & - & $\mathrm{X}$ \\
\hline *Ortilia dicoma(Hewitson, 1864) & - & $\mathrm{X}$ & - & - & - & $\mathrm{X}$ \\
\hline Ortilia ithra (Kirby, 1900) & - & - & $\mathrm{X}$ & - & - & - \\
\hline Ortilia orthia (Hewitson, 1864) & $\mathrm{X}$ & $\mathrm{X}$ & $\mathrm{X}$ & $\mathrm{X}$ & - & $\mathrm{X}$ \\
\hline *Siproeta epaphus trayja Hübner, 1823 & - & - & $\mathrm{X}$ & $\mathrm{X}$ & - & $\mathrm{X}$ \\
\hline *Siproeta stelenes meridionalis (Fruhstorfer, 1909) & - & - & $\mathrm{X}$ & - & - & - \\
\hline *Smyrna blomfildia (Fabricius, 1781) & $\mathrm{X}$ & - & $\mathrm{X}$ & - & - & - \\
\hline Tegosa claudina (Eschscholtz, 1821) & $\mathrm{X}$ & $\mathrm{X}$ & $\mathrm{X}$ & $\mathrm{X}$ & $X$ & $\mathrm{X}$ \\
\hline *Tegosa orobia (Hewitson, 1864) & $\mathrm{X}$ & $\mathrm{X}$ & $X$ & - & $\mathrm{X}$ & $\mathrm{X}$ \\
\hline Telenassa teletusa (Godart, 1824) & $X$ & $X$ & $X$ & $X$ & $X$ & $X$ \\
\hline Vanessa braziliensis (Moore, 1883) & $\mathrm{X}$ & $\mathrm{X}$ & $\mathrm{X}$ & $\mathrm{X}$ & $\mathrm{X}$ & $\mathrm{X}$ \\
\hline Vanessa myrinna (Doubleday, 1849) & - & - & $X$ & - & - & - \\
\hline \multicolumn{7}{|l|}{ Limenitidinae $(S=7)$} \\
\hline Adelpha hyas (Doyère, 1840) & $\mathrm{X}$ & $X$ & $\mathrm{X}$ & $X$ & - & $\mathrm{X}$ \\
\hline *Adelpha lycorias (Godart, 1824) & - & - & - & $\mathrm{X}$ & - & - \\
\hline Adelpha mythra (Godart, 1824) & $X$ & $\mathrm{X}$ & $X$ & $\mathrm{X}$ & - & - \\
\hline *Adelpha serpa (Boisduval, 1836) & $\mathrm{X}$ & - & $X$ & - & - & - \\
\hline Adelpha syma (Godart, 1824) & $\mathrm{X}$ & $\mathrm{X}$ & $\mathrm{X}$ & $\mathrm{X}$ & - & $\mathrm{X}$ \\
\hline *Adelpha thessalia indefecta Fruhstorfer, 1913 & - & - & $X$ & - & - & - \\
\hline *Adelpha zea (Hewitson, 1850) & $\mathrm{X}$ & - & $\mathrm{X}$ & - & - & - \\
\hline \multicolumn{7}{|l|}{ Heliconiinae $(\mathrm{S}=21)$} \\
\hline *Actinote alalia (Felder \& Felder, 1860) & - & - & $\mathrm{X}$ & $X$ & - & - \\
\hline Actinote carycina Jordan, 1913 & $\mathrm{X}$ & $\mathrm{X}$ & $X$ & $X$ & $\mathrm{X}$ & $\mathrm{X}$ \\
\hline Actinote catarina Penz, 1996 & - & - & $X$ & - & - & - \\
\hline *Actinote discrepans D’Almeida, 1958 & - & - & $\mathrm{X}$ & - & - & - \\
\hline *Actinote mamita (Burmeister, 1861) & - & - & - & - & - & $\mathrm{X}$ \\
\hline Actinote melanisans Oberthür, 1917 & $\mathrm{X}$ & $\mathrm{X}$ & $\mathrm{X}$ & $\mathrm{X}$ & $\mathrm{X}$ & $\mathrm{X}$ \\
\hline *Actinote parapheles Jordan, 1913 & - & - & $\mathrm{X}$ & $X$ & - & - \\
\hline Actinote surima (Schaus, 1902) & - & $\mathrm{X}$ & $X$ & - & - & $\mathrm{X}$ \\
\hline Actinote thalia pyrrha (Fabricius, 1775) & - & $\mathrm{X}$ & $X$ & $\mathrm{X}$ & - & - \\
\hline Agraulis vanillae maculosa (Stichel, 1908) & - & $\mathrm{X}$ & $X$ & - & $X$ & $\mathrm{X}$ \\
\hline Dione juno juno (Cramer, 1779) & $\mathrm{X}$ & - & $X$ & - & - & - \\
\hline Dione moneta moneta Hübner, 1825 & - & - & $X$ & - & - & - \\
\hline Dryas iulia alcionea (Cramer, 1779) & $\mathrm{X}$ & $X$ & $X$ & $\mathrm{X}$ & - & $\mathrm{X}$ \\
\hline *Eueides aliphera aliphera (Godart, 1819) & $\mathrm{X}$ & - & - & - & - & - \\
\hline Eueides isabella dianasa (Hübner, 1806) & - & $X$ & - & - & - & - \\
\hline *Euptoieta claudia (Cramer, 1775) & - & - & $\mathrm{X}$ & - & - & $\mathrm{X}$ \\
\hline
\end{tabular}

Lista de espécies de borboletas registradas de março de 2006 a maio de 2008 na Floresta Nacional de São Francisco de Paula e entorno. MN, mata nativa; NR, mata nativa mesclada com reflorestamento de Araucária; RA, reflorestamento de Araucária de mata aberta; RF, Reflorestamento de Araucária de mata fechada; RP, reflorestamento de Pinus; CN, Campo nativo; S, riqueza de espécies. * novos registros para a região dos Campos de Cima da Serra. 
Tabela 1. Continuação...

\begin{tabular}{|c|c|c|c|c|c|c|}
\hline \multirow[t]{2}{*}{ Famílias/Subfamílias/Espécies } & \multicolumn{6}{|c|}{ Ambientes } \\
\hline & MN & NR & RA & RF & $\mathbf{R P}$ & $\mathbf{C N}$ \\
\hline Heliconius besckei Ménétriés, 1857 & $\mathrm{X}$ & $\mathrm{X}$ & $\mathrm{X}$ & $\mathrm{X}$ & $\mathrm{X}$ & - \\
\hline Heliconius erato phyllis (Fabricius, 1775) & $\mathrm{X}$ & $\mathrm{X}$ & $X$ & $\mathrm{X}$ & $\mathrm{X}$ & $\mathrm{X}$ \\
\hline Heliconius ethilla narcaea Godart, 1819 & $\mathrm{X}$ & $\mathrm{X}$ & $\mathrm{X}$ & $X$ & $\mathrm{X}$ & - \\
\hline *Heliconius sara apseudes (Hübner, 1813) & - & - & $\mathrm{X}$ & - & - & - \\
\hline Philaethria wernickei (Röber, 1906) & $\mathrm{X}$ & $\mathrm{X}$ & $\mathrm{X}$ & $\mathrm{X}$ & - & $\mathrm{X}$ \\
\hline \multicolumn{7}{|l|}{ PIERIDAE $(S=26)$} \\
\hline \multicolumn{7}{|l|}{ Dismorphiinae $(S=7)$} \\
\hline Dismorphia astyocha Hübner, 1831 & - & - & $X$ & $\mathrm{X}$ & - & - \\
\hline Dismorphia crisia (Drury, 1782) & - & $\mathrm{X}$ & $\mathrm{X}$ & $\mathrm{X}$ & - & - \\
\hline Dismorphia melia (Godart, 1824) & $\mathrm{X}$ & $\mathrm{X}$ & $\mathrm{X}$ & $\mathrm{X}$ & - & - \\
\hline Dismorphia thermesia (Godart, 1819) & $\mathrm{X}$ & $\mathrm{X}$ & $X$ & $\mathrm{X}$ & $\mathrm{X}$ & - \\
\hline Enantia lina psamathe (Fabricius, 1793) & - & $\mathrm{X}$ & $X$ & $\mathrm{X}$ & - & - \\
\hline Enantia melite (Linnaeus, 1763) & - & $\mathrm{X}$ & $X$ & $\mathrm{X}$ & - & $X$ \\
\hline Pseudopieris nehemia (Boisduval, 1836) & - & $X$ & $X$ & $\mathrm{X}$ & $X$ & $X$ \\
\hline \multicolumn{7}{|l|}{ Coliadinae $(\mathrm{S}=11)$} \\
\hline Colias lesbia lesbia (Fabricius, 1775) & - & - & - & - & - & $\mathrm{X}$ \\
\hline Eurema albula sinoe (Godart, 1819) & - & - & $\mathrm{X}$ & - & - & - \\
\hline Eurema deva deva (Doubleday, 1847) & - & - & $\mathrm{X}$ & - & - & - \\
\hline Eurema phiale paula (Röber, 1909) & - & - & $\mathrm{X}$ & - & $\mathrm{X}$ & $\mathrm{X}$ \\
\hline Phoebis argante argante (Fabricius, 1775) & - & $\mathrm{X}$ & $\mathrm{X}$ & $\mathrm{X}$ & - & $\mathrm{X}$ \\
\hline Phoebis neocypris neocypris (Hübner, 1823) & $\mathrm{X}$ & $\mathrm{X}$ & $\mathrm{X}$ & $\mathrm{X}$ & $\mathrm{X}$ & $\mathrm{X}$ \\
\hline Phoebis philea philea (Linneaus, 1763) & $\mathrm{X}$ & - & $\mathrm{X}$ & - & - & - \\
\hline Phoebis sennae marcellina (Cramer, 1777) & - & - & $\mathrm{X}$ & $\mathrm{X}$ & - & - \\
\hline Pyrisitia nise tenella (Boisduval,1836) & - & - & $X$ & $\mathrm{X}$ & - & - \\
\hline Pyrisitia leuce leuce (Boisduval, 1836) & - & $\mathrm{X}$ & $\mathrm{X}$ & - & $\mathrm{X}$ & $X$ \\
\hline Rhabdodryas trite banksi Breyer, 1939 & $\mathrm{X}$ & $\mathrm{X}$ & $\mathrm{X}$ & - & - & $\mathrm{X}$ \\
\hline \multicolumn{7}{|l|}{ Pierinae $(S=8)$} \\
\hline Ascia monuste orseis (Godart, 1819) & - & - & $\mathrm{X}$ & - & - & - \\
\hline Catasticta bithys (Hübner, 1831) & - & - & - & $X$ & - & - \\
\hline Glutophrissa drusilla (Cramer, 1777) & - & - & $\mathrm{X}$ & $X$ & - & - \\
\hline Hesperocharis erota (Lucas, 1852) & $\mathrm{X}$ & $\mathrm{X}$ & $\mathrm{X}$ & $X$ & $X$ & $X$ \\
\hline Hesperocharis paranensis Schaus, 1898 & - & - & $\mathrm{X}$ & $X$ & - & $\mathrm{X}$ \\
\hline Leptophobia aripa balidia (Boisduval, 1836) & - & - & $\mathrm{X}$ & - & - & - \\
\hline Pereute swainsoni (Gray, 1832) & - & - & $\mathrm{X}$ & - & - & $\mathrm{X}$ \\
\hline Theochila maenacte (Boisduval, 1836) & $\mathrm{X}$ & $\mathrm{X}$ & $\mathrm{X}$ & $\mathrm{X}$ & $\mathrm{X}$ & $\mathrm{X}$ \\
\hline \multicolumn{7}{|l|}{ PAPILIONIDAE $(\mathrm{S}=14)$} \\
\hline \multicolumn{7}{|l|}{ Papilioninae $(S=14)$} \\
\hline Battus polydamas polydamas (Linnaeus, 1758) & - & - & $\mathrm{X}$ & $\mathrm{X}$ & - & $X$ \\
\hline Battus polystictus polystictus (Butler, 1874) & - & $\mathrm{X}$ & - & $\mathrm{X}$ & - & - \\
\hline Heraclides anchisiades capys (Hübner, 1809) & - & - & $\mathrm{X}$ & $\mathrm{X}$ & - & - \\
\hline Heraclides astyalus astyalus (Godart, 1819) & - & $\mathrm{X}$ & $\mathrm{X}$ & $\mathrm{X}$ & - & $\mathrm{X}$ \\
\hline Heraclides hectorides (Esper, 1794) & $X$ & $\mathrm{X}$ & $\mathrm{X}$ & $\mathrm{X}$ & $X$ & $\mathrm{X}$ \\
\hline Heraclides thoas brasiliensis (Rothschild \& Jordan, 1906) & - & $\mathrm{X}$ & $X$ & $\mathrm{X}$ & - & - \\
\hline Mimoides lysithous eupatorion (Lucas, 1859) & - & $X$ & $X$ & $\mathrm{X}$ & - & - \\
\hline Mimoides lysithous rurik (Eschscholtz, 1821) & $\mathrm{X}$ & $\mathrm{X}$ & $X$ & $\mathrm{X}$ & - & - \\
\hline Parides agavus (Drury, 1782) & $\mathrm{X}$ & - & - & - & - & - \\
\hline Parides anchises nephalion (Godart, 1819) & - & $\mathrm{X}$ & $X$ & $\mathrm{X}$ & - & - \\
\hline Protesilaus helios (Rothschild \& Jordan, 1906) & - & - & $\mathrm{X}$ & - & - & $\mathrm{X}$ \\
\hline
\end{tabular}

Lista de espécies de borboletas registradas de março de 2006 a maio de 2008 na Floresta Nacional de São Francisco de Paula e entorno. MN, mata nativa; NR, mata nativa mesclada com reflorestamento de Araucária; RA, reflorestamento de Araucária de mata aberta; RF, Reflorestamento de Araucária de mata fechada; RP, reflorestamento de Pinus; CN, Campo nativo; S, riqueza de espécies. * novos registros para a região dos Campos de Cima da Serra. 
Tabela 1. Continuação...

\begin{tabular}{|c|c|c|c|c|c|c|}
\hline \multirow[t]{2}{*}{ Famílias/Subfamílias/Espécies } & \multicolumn{6}{|c|}{ Ambientes } \\
\hline & MN & NR & RA & RF & $\mathbf{R P}$ & CN \\
\hline Protesilaus protesilaus nigricornis (Staudinger, 1884) & - & - & $\mathrm{X}$ & - & - & - \\
\hline Pterourus menatius cleotas (Gray, 1832) & - & - & - & $\mathrm{X}$ & - & - \\
\hline Pterourus scamander scamander (Boisduval, 1836) & - & - & $\mathrm{X}$ & $\mathrm{X}$ & - & $\mathrm{X}$ \\
\hline \multicolumn{7}{|l|}{ HESPERIIDAE $(S=82)$} \\
\hline \multicolumn{7}{|l|}{ Pyrrhopyginae $(\mathrm{S}=5)$} \\
\hline Elbella adonis (Bell, 1931) & - & - & - & $\mathrm{X}$ & - & - \\
\hline Elbella mariae mariae (Bell, 1931) & - & - & $\mathrm{X}$ & - & - & - \\
\hline Mysoria barcastus barta Evans, 1951 & $\mathrm{X}$ & - & - & - & - & - \\
\hline Pyrrhopige charybdis charybdis Westwood, 1852 & $\mathrm{X}$ & $\mathrm{X}$ & - & - & - & - \\
\hline Sarbia damippe Mabille \& Boullet, 1908 & - & $\mathrm{X}$ & $\mathrm{X}$ & $\mathrm{X}$ & - & $\mathrm{X}$ \\
\hline \multicolumn{7}{|l|}{ Pyrginae $(S=29)$} \\
\hline Achlyodes busirus rioja Evans, 1953 & $\mathrm{X}$ & $\mathrm{X}$ & $\mathrm{X}$ & $\mathrm{X}$ & - & $\mathrm{X}$ \\
\hline Achlyodes mithridates thraso (Hübner, 1807) & - & - & $\mathrm{X}$ & $\mathrm{X}$ & - & - \\
\hline Anisochoria sublimbata Mabille, 1883 & - & - & $\mathrm{X}$ & $\mathrm{X}$ & - & - \\
\hline Astraptes erycina (Plötz, 1881) & - & - & $\mathrm{X}$ & $\mathrm{X}$ & - & - \\
\hline Astraptes fulgerator fulgerator (Walch, 1775) & $\mathrm{X}$ & - & $X$ & $\mathrm{X}$ & - & - \\
\hline Astraptes naxos (Hewitson, 1867) & - & $\mathrm{X}$ & $\mathrm{X}$ & $\mathrm{X}$ & - & - \\
\hline Autochton integrifascia (Mabille, 1891) & - & $\mathrm{X}$ & - & $X$ & - & - \\
\hline Autochton zarex (Hübner, 1818) & - & $X$ & $X$ & $X$ & - & - \\
\hline Celaenorrhinus eligius punctiger (Burmeister, 1878) & $\mathrm{X}$ & $X$ & $X$ & $X$ & - & $\mathrm{X}$ \\
\hline Gorgythion begga begga (Prittwitz, 1868) & $X$ & - & $X$ & $\mathrm{X}$ & - & - \\
\hline Helias phalaenoides palpalis (Latreille, 1824) & - & - & - & $\mathrm{X}$ & - & - \\
\hline Heliopetes alana (Reakirt,1868) & - & - & $\mathrm{X}$ & - & - & - \\
\hline Heliopetes arsalte (Linnaeus, 1758) & - & - & $\mathrm{X}$ & - & - & $\mathrm{X}$ \\
\hline Heliopetes libra Evans, 1944 & - & - & $\mathrm{X}$ & - & - & - \\
\hline Heliopetes leucola (Hewitson, 1868) & - & - & $X$ & - & - & - \\
\hline Heliopetes omrina (Butler, 1870) & - & - & $\mathrm{X}$ & $\mathrm{X}$ & - & - \\
\hline Heliopetes purgia Schaus, 1902 & - & - & $X$ & - & - & - \\
\hline Milanion leucaspis (Mabille, 1878) & - & $\mathrm{X}$ & $\mathrm{X}$ & $\mathrm{X}$ & - & - \\
\hline Ocella monophthalma (Plotz, 1884) & - & - & - & $\mathrm{X}$ & - & - \\
\hline Phocides pialia pialia (Hewitson, 1857) & - & $\mathrm{X}$ & $\mathrm{X}$ & - & - & - \\
\hline Pyrgus orcus (Stoll, 1780) & $\mathrm{X}$ & - & $X$ & - & - & - \\
\hline Pythonides lancea (Hewitson, 1868) & $\mathrm{X}$ & $\mathrm{X}$ & $\mathrm{X}$ & $\mathrm{X}$ & - & - \\
\hline Quadrus cerialis (Stoll, 1782) & - & - & $X$ & - & - & - \\
\hline Staphylus sp. & $\mathrm{X}$ & - & $\mathrm{X}$ & $\mathrm{X}$ & - & - \\
\hline Theagenes dichrous (Mabille, 1878) & $\mathrm{X}$ & - & $\mathrm{X}$ & $\mathrm{X}$ & - & - \\
\hline Trina geometrina geometrina (Felder \& Felder, 1867) & - & $X$ & - & $\mathrm{X}$ & - & - \\
\hline Urbanus dorantes (Stoll, 1790) & - & - & $\mathrm{X}$ & - & - & - \\
\hline Urbanus esta Evans, 1952 & - & - & $\mathrm{X}$ & - & - & - \\
\hline Urbanus teleus (Hübner, 1821) & $X$ & $X$ & $X$ & $\mathrm{X}$ & $\mathrm{X}$ & $\mathrm{X}$ \\
\hline \multicolumn{7}{|l|}{ Heteropterinae $(\mathrm{S}=1)$} \\
\hline Dardarina aspila Mielke, 1966 & - & - & $\mathrm{X}$ & $\mathrm{X}$ & - & - \\
\hline \multicolumn{7}{|l|}{ Hesperiinae $(S=47)$} \\
\hline Ancyloxypha nitedula (Burmeister, 1878) & - & - & - & - & - & $\mathrm{X}$ \\
\hline Anthoptus epictetus (Fabricius, 1793) & $\mathrm{X}$ & $\mathrm{X}$ & $X$ & $X$ & $\mathrm{X}$ & - \\
\hline Appia appia Evans, 1955 & - & - & - & - & - & $\mathrm{X}$ \\
\hline Arotis derasa brunnea (Mielke, 1972) & - & - & $X$ & - & - & - \\
\hline Callimormus interpunctata (Plötz, 1884) & - & $\mathrm{X}$ & $\mathrm{X}$ & $\mathrm{X}$ & - & - \\
\hline
\end{tabular}

Lista de espécies de borboletas registradas de março de 2006 a maio de 2008 na Floresta Nacional de São Francisco de Paula e entorno. MN, mata nativa; NR, mata nativa mesclada com reflorestamento de Araucária; RA, reflorestamento de Araucária de mata aberta; RF, Reflorestamento de Araucária de mata fechada; RP, reflorestamento de Pinus; CN, Campo nativo; S, riqueza de espécies. * novos registros para a região dos Campos de Cima da Serra. 
Tabela 1. Continuação...

\begin{tabular}{|c|c|c|c|c|c|c|}
\hline \multirow[t]{2}{*}{ Famílias/Subfamílias/Espécies } & \multicolumn{6}{|c|}{ Ambientes } \\
\hline & MN & NR & $\mathbf{R A}$ & $\mathbf{R F}$ & $\mathbf{R P}$ & $\mathbf{C N}$ \\
\hline Callimormus rivera (Plötz, 1882) & $\mathrm{X}$ & $\mathrm{X}$ & $\mathrm{X}$ & $\mathrm{X}$ & $\mathrm{X}$ & $\mathrm{X}$ \\
\hline Cobalopsis nero (Herrich-Schäffer, 1869) & - & $\mathrm{X}$ & - & - & - & - \\
\hline Cobalopsis vorgia (Schaus, 1902) & - & - & - & - & $\mathrm{X}$ & - \\
\hline Conga iheringii (Mabille, 1891) & $\mathrm{X}$ & $\mathrm{X}$ & $\mathrm{X}$ & $\mathrm{X}$ & $\mathrm{X}$ & $\mathrm{X}$ \\
\hline Conga immaculata (Bell, 1930) & $\mathrm{X}$ & - & $\mathrm{X}$ & $\mathrm{X}$ & - & - \\
\hline Conga zela (Plotz, 1883) & - & - & - & - & $\mathrm{X}$ & - \\
\hline Copaeodes jean favor Evans, 1955 & - & - & - & - & - & $\mathrm{X}$ \\
\hline Corticea immocerinus (Hayward, 1934) & - & - & - & - & $\mathrm{X}$ & - \\
\hline Corticea lysias potex Evans, 1955 & $\mathrm{X}$ & $\mathrm{X}$ & - & - & - & $\mathrm{X}$ \\
\hline Corticea obscura Mielke, 1969 & - & - & $\mathrm{X}$ & $\mathrm{X}$ & - & $\mathrm{X}$ \\
\hline Corticea sp. & - & - & - & $\mathrm{X}$ & - & - \\
\hline Cumbre cumbre (Schaus, 1902) & $\mathrm{X}$ & - & - & $\mathrm{X}$ & - & - \\
\hline Cymaenes distigma (Plötz, 1882) & - & - & - & $\mathrm{X}$ & - & - \\
\hline Cymaenes tripunctata (Latreille, 1824) & - & - & $\mathrm{X}$ & - & - & - \\
\hline Enosis schausi Mielke \& Casagrande, 2002 & - & - & - & $\mathrm{X}$ & - & - \\
\hline Hylephyla ancora (Plötz, 1883) & - & - & - & - & - & $\mathrm{X}$ \\
\hline Lamponia lamponia (Hewitson, 1876) & - & $\mathrm{X}$ & $\mathrm{X}$ & $\mathrm{X}$ & - & - \\
\hline Lucida lucia lucia (Capronnier, 1874) & - & - & - & $\mathrm{X}$ & - & - \\
\hline Lucida ranesus (Schaus, 1902) & - & - & $\mathrm{X}$ & - & - & - \\
\hline Lychnuchoides ozias ozias (Hewitson, 1878) & - & $\mathrm{X}$ & - & - & - & - \\
\hline Lychnuchus celsus (Fabricius, 1793) & - & - & - & $\mathrm{X}$ & - & - \\
\hline Miltomiges cinnamomea (Herrich-Schäffer, 1869) & $\mathrm{X}$ & $\mathrm{X}$ & $\mathrm{X}$ & - & - & - \\
\hline Mnasitheus ritans (Schaus, 1902) & $\mathrm{X}$ & $\mathrm{X}$ & $\mathrm{X}$ & $\mathrm{X}$ & - & - \\
\hline Moeris striga striga (Geyer, 1832) & - & $\mathrm{X}$ & - & - & - & - \\
\hline Nastra lurida (Herrich-Schäffer, 1869) & $\mathrm{X}$ & $\mathrm{X}$ & $\mathrm{X}$ & $\mathrm{X}$ & $\mathrm{X}$ & - \\
\hline Nyctelius nyctelius nyctelius (Latreille, 1824) & - & - & $\mathrm{X}$ & - & - & - \\
\hline Perichares philetes aurina Evans, 1955 & - & $\mathrm{X}$ & - & - & - & - \\
\hline Pompeius pompeius (Latreille, 1824) & - & $\mathrm{X}$ & $\mathrm{X}$ & - & - & - \\
\hline Psoralis stacara (Schaus, 1902) & - & $\mathrm{X}$ & - & - & - & - \\
\hline Remella remus (Fabricius, 1798) & - & $\mathrm{X}$ & - & - & - & - \\
\hline Saniba sabina (Plötz, 1882) & - & $\mathrm{X}$ & - & $\mathrm{X}$ & - & - \\
\hline Sodalia coler (Schaus, 1902) & $\mathrm{X}$ & $\mathrm{X}$ & $\mathrm{X}$ & $\mathrm{X}$ & - & - \\
\hline Sucova sucova (Schaus, 1902) & - & - & $\mathrm{X}$ & - & - & - \\
\hline Thespieus catochra (Plötz, 1882) & - & - & - & - & - & $\mathrm{X}$ \\
\hline Thespieus himella (Hewitson, 1868) & - & - & $\mathrm{X}$ & - & - & - \\
\hline Thespieus jora Evans, 1955 & - & - & - & $\mathrm{X}$ & - & - \\
\hline Vehilius clavicula (Plötz, 1884) & $\mathrm{X}$ & $\mathrm{X}$ & $\mathrm{X}$ & $\mathrm{X}$ & - & - \\
\hline Vehilius inca (Scudder, 1872) & - & - & $\mathrm{X}$ & - & - & - \\
\hline Vehilius stictomenes stictomenes (Butler,1877) & - & - & $\mathrm{X}$ & - & - & - \\
\hline Vinius letis (Plötz, 1883) & - & $\mathrm{X}$ & - & - & - & - \\
\hline Virga riparia Mielke, 1969 & - & - & - & - & - & $\mathrm{X}$ \\
\hline Zariaspes mys (Hübner, 1808) & - & $\mathrm{X}$ & $\mathrm{X}$ & $\mathrm{X}$ & - & $\mathrm{X}$ \\
\hline \multicolumn{7}{|l|}{ LYCAENIDAE $(\mathrm{S}=31)$} \\
\hline \multicolumn{7}{|l|}{ Theclinae $(\mathrm{S}=29)$} \\
\hline Arawacus binangula (Schaus, 1902) & - & - & $\mathrm{X}$ & - & - & - \\
\hline Arawacus ellida (Hewitson, 1867) & - & $\mathrm{X}$ & - & - & - & - \\
\hline Arawacus meliboeus (Fabricius, 1793) & $\mathrm{X}$ & $\mathrm{X}$ & $\mathrm{X}$ & $\mathrm{X}$ & $\mathrm{X}$ & $\mathrm{X}$ \\
\hline Arawacus separata (Lathy, 1926) & - & - & $\mathrm{X}$ & - & $\mathrm{X}$ & - \\
\hline
\end{tabular}

Lista de espécies de borboletas registradas de março de 2006 a maio de 2008 na Floresta Nacional de São Francisco de Paula e entorno. MN, mata nativa; NR, mata nativa mesclada com reflorestamento de Araucária; RA, reflorestamento de Araucária de mata aberta; RF, Reflorestamento de Araucária de mata fechada; RP, reflorestamento de Pinus; CN, Campo nativo; S, riqueza de espécies. * novos registros para a região dos Campos de Cima da Serra. 
Tabela 1. Continuação...

\begin{tabular}{|c|c|c|c|c|c|c|}
\hline \multirow[t]{2}{*}{ Famílias/Subfamílias/Espécies } & \multicolumn{6}{|c|}{ Ambientes } \\
\hline & MN & NR & $\mathbf{R A}$ & $\mathbf{R F}$ & $\mathbf{R P}$ & $\mathbf{C N}$ \\
\hline Arawacus tadita (Hewitson, 1877) & - & $\mathrm{X}$ & $\mathrm{X}$ & - & - & - \\
\hline Brevianta celelata (Hewitson, 1874) & - & - & - & - & - & $\mathrm{X}$ \\
\hline Calycopis caulonia (Hewitson, 1877) & - & $\mathrm{X}$ & $\mathrm{X}$ & $\mathrm{X}$ & - & $\mathrm{X}$ \\
\hline Contrafacia imma (Prittwitz, 1865) & - & $\mathrm{X}$ & $\mathrm{X}$ & $\mathrm{X}$ & - & - \\
\hline Cyanophrys acaste (Prittwitz,1865) & - & $\mathrm{X}$ & - & $\mathrm{X}$ & - & - \\
\hline Cyanophrys remus (Hewitson, 1868) & $\mathrm{X}$ & $\mathrm{X}$ & $\mathrm{X}$ & $\mathrm{X}$ & - & - \\
\hline Denivia curitibaensis (K. Johnson, 1992) & - & - & - & $\mathrm{X}$ & - & - \\
\hline Denivia deniva (Hewitson, 1874) & - & - & $\mathrm{X}$ & $\mathrm{X}$ & - & $\mathrm{X}$ \\
\hline Dicyaca eumorpha (Hayward, 1949) & - & - & $\mathrm{X}$ & - & - & - \\
\hline Erora gabina (Godman \& Salvin, 1887) & - & - & $\mathrm{X}$ & - & - & - \\
\hline Gargina caninius (H.H. Druce, 1907) & - & $\mathrm{X}$ & - & - & - & - \\
\hline Hypostrymon sp. & - & $\mathrm{X}$ & - & - & - & - \\
\hline Lamprospilus nubilum (H. H. Druce, 1907) & $\mathrm{X}$ & - & $\mathrm{X}$ & $\mathrm{X}$ & - & - \\
\hline Laothus phydela (Hewitson, 1867) & - & $\mathrm{X}$ & $\mathrm{X}$ & $\mathrm{X}$ & $\mathrm{X}$ & - \\
\hline Ocaria thales(Fabricius, 1793) & $\mathrm{X}$ & - & - & - & - & - \\
\hline Parrhasius selika (Hewitson, 1874) & $\mathrm{X}$ & - & - & - & - & - \\
\hline Rekoa malina (Hewitson, 1867) & - & $\mathrm{X}$ & $\mathrm{X}$ & - & - & - \\
\hline Siderus philinna (Hewitson, 1868) & - & $\mathrm{X}$ & - & - & - & - \\
\hline Strymon bazochii (Godart, 1824) & - & - & $\mathrm{X}$ & - & - & - \\
\hline Strymon lucena (Hewitson, 1868) & - & - & $\mathrm{X}$ & - & - & - \\
\hline Strymon oreala (Hewitson, 1868) & - & - & $\mathrm{X}$ & - & - & - \\
\hline Thaeides theia (Hewitson, 1870) & $\mathrm{X}$ & - & - & - & - & - \\
\hline Theritas hemon (Cramer, 1775) & - & - & - & $\mathrm{X}$ & - & - \\
\hline Theritas triquetra (Hewitson, 1865) & - & $\mathrm{X}$ & $\mathrm{X}$ & $\mathrm{X}$ & $\mathrm{X}$ & $\mathrm{X}$ \\
\hline Tmolus echion (Linnaeus, 1767) & - & - & $\mathrm{X}$ & - & - & - \\
\hline \multicolumn{7}{|l|}{ Polyommatinae $(\mathrm{S}=2)$} \\
\hline Leptotes cassius (Cramer, 1775) & - & - & $\mathrm{X}$ & - & - & - \\
\hline Zizula cyna (H. H. Edwards, 1881) & - & - & $\mathrm{X}$ & - & - & - \\
\hline \multicolumn{7}{|l|}{ RIODINIDAE ( $\mathrm{S}=16)$} \\
\hline \multicolumn{7}{|l|}{ Euselasiinae $(\mathrm{S}=1)$} \\
\hline Euselasia euploea (Hewitson, 1855) & - & $\mathrm{X}$ & $\mathrm{X}$ & - & - & - \\
\hline \multicolumn{7}{|l|}{ Riodininae $(S=15)$} \\
\hline Barbicornis basilis mona Westwood, 1851 & - & - & $\mathrm{X}$ & - & - & - \\
\hline Calephelis nr. braziliensis McAlpine, 1971 & - & - & $X$ & - & - & - \\
\hline Charis cadytis Hewitson, 1866 & $\mathrm{X}$ & $\mathrm{X}$ & $\mathrm{X}$ & $X$ & - & $\mathrm{X}$ \\
\hline Dachetola azora (Godart, 1824) & $\mathrm{X}$ & - & $\mathrm{X}$ & $\mathrm{X}$ & - & $\mathrm{X}$ \\
\hline Emesis melancholica Stichel, 1916 & $\mathrm{X}$ & $\mathrm{X}$ & $\mathrm{X}$ & $\mathrm{X}$ & - & - \\
\hline Harveyope argiella (H.W. Bates, 1868) & - & $\mathrm{X}$ & $\mathrm{X}$ & - & - & - \\
\hline Harveyope sejuncta (Stichel, 1910) & - & - & $\mathrm{X}$ & $\mathrm{X}$ & - & - \\
\hline Ithomiola nepos (Fabricius, 1793) & - & $\mathrm{X}$ & $\mathrm{X}$ & - & - & - \\
\hline Lasaia agesilas (Latreille, 1809) & - & - & $\mathrm{X}$ & - & - & - \\
\hline Melanis smithiae (Westwood, 1851) & $\mathrm{X}$ & - & - & - & - & - \\
\hline Pirascca sagaris phyrgiana (Stichel, 1916) & $\mathrm{X}$ & - & $\mathrm{X}$ & - & - & - \\
\hline Pseudotinea $\mathrm{cf}$. hemis(Schaus, 1927) & - & - & $X$ & $\mathrm{X}$ & - & - \\
\hline Stichelia bocchoris (Hewitson, 1876) & $\mathrm{X}$ & $\mathrm{X}$ & $\mathrm{X}$ & $\mathrm{X}$ & $\mathrm{X}$ & - \\
\hline Synargis axenus (Hewitson, 1876) & - & - & - & - & - & $\mathrm{X}$ \\
\hline Synargis paulistina(Stichel, 1910) & - & - & $\mathrm{X}$ & $\mathrm{X}$ & $\mathrm{X}$ & - \\
\hline
\end{tabular}

Lista de espécies de borboletas registradas de março de 2006 a maio de 2008 na Floresta Nacional de São Francisco de Paula e entorno. MN, mata nativa; NR, mata nativa mesclada com reflorestamento de Araucária; RA, reflorestamento de Araucária de mata aberta; RF, Reflorestamento de Araucária de mata fechada; RP, reflorestamento de Pinus; CN, Campo nativo; S, riqueza de espécies. * novos registros para a região dos Campos de Cima da Serra. 
Iserhard, C.A. et al.

Tabela 2. Representatividade das famílias de borboletas para diferentes localidades e regiões.

Table 2. Percentage composition of butterfly families for different areas and regions.

\begin{tabular}{lcccc}
\hline & Sul América do Sul & Brasil & Maquiné & \multirow{2}{*}{ FOM-CCS } \\
\cline { 2 - 4 } & Mor & B\&F & I\&R & 39 \\
\hline Nymphalidae & 28,1 & 24 & 36 & 29,6 \\
Hesperiidae & 41,3 & 35,5 & 33 & $17 *$ \\
Lycaenidae & 22 & 36 & 8 & 9,4 \\
Pieridae & 5,2 & 2 & 4 & 5 \\
Papilionidae & 3,4 & 2,5 & 4 & 5 \\
\hline
\end{tabular}

Riqueza de espécies por família (\%) em assembléias de borboletas no sul da América do Sul, Brasil, Vale do rio Maquiné (Maquiné) e Floresta Ombrófila Mista-Campos de Cima da Serra (FOM-CCS). (B\&F) Brown \& Freitas (1999), (I\&R) Iserhard \& Romanowski (2004), (Mor), Morais et al. (2007). *

Riodinidae incluído dentro de Lycaenidae

Tabela 3. Novos registros de borboletas para o Rio Grande do Sul, Brasil.

Table 3. New occurrences of butterflies in Rio Grande do Sul State, Brazil.

\begin{tabular}{|c|c|c|}
\hline Famílias/Subfamílias & Espécies & Data da primeira coleta \\
\hline \multicolumn{3}{|l|}{ NYMPHALIDAE } \\
\hline Ithomiinae & Hyalenna pascua (Schaus, 1902) & 20.v.2007 \\
\hline \multirow[t]{2}{*}{ Satyrinae } & Euptychoides castrensis (Schaus, 1902) & 26.iii.2006 \\
\hline & Yphthimoides viviana (Romieux, 1927) & 08.iii.2008 \\
\hline Heliconiinae & Actinote alalia (Felder \& Felder, 1860) & 03.ii.2007 \\
\hline \multicolumn{3}{|l|}{ HESPERIIDAE } \\
\hline Pyrginae & Ocella monophthalma (Plötz, 1884) & 03.ii.2008 \\
\hline \multirow[t]{2}{*}{ Hesperiinae } & Arotis derasa brunnea (Mielke, 1972) & 31.xii.2007 \\
\hline & Enosis schausi (Mielke \& Casagrande, 2002) & 02.ii.2007 \\
\hline \multicolumn{3}{|l|}{ LYCAENIDAE } \\
\hline \multirow[t]{3}{*}{ Theclinae } & Erora gabina (Godman \& Salvin, 1987) & 11.xii.2006 \\
\hline & Gargina caninius (H.H. Druce, 1907) & 26.v.2007 \\
\hline & Siderus philinna (Hewitson, 1868) & 01.i.2008 \\
\hline \multicolumn{3}{|l|}{ RIODINIDAE } \\
\hline \multirow[t]{3}{*}{ Riodininae } & Harveyope sejuncta (Stichel, 1910) & 12.xii.2006 \\
\hline & Pseudotinea hemis (Schaus, 1927) & 11.xii.2006 \\
\hline & Synargis paulistina (Stichel, 1910) & 31.xii. 2007 \\
\hline
\end{tabular}

Novos registros de espécies de borboletas, com as datas da primeira coleta, para o Rio Grande do Sul, registradas entre março de 2006 e maio de 2008 na Floresta Nacional de São Francisco de Paula e entorno.

Duas espécies de Pieridae, Dismorphia crisia (Drury, 1782) e Dismorphia melia (Godart, 1824) e uma de Nymphalidae, Hyalenna pascua (Schaus, 1902), são consideradas indicadoras de ambientes em boas condições de preservação (Brown \& Freitas 2000a). Dismorphia melia foi registrada 26 vezes, enquanto $D$. crisia foi registrada apenas seis vezes. Estavam associadas tanto a trilhas com reflorestamento de araucária quanto à mata nativa. Estas duas espécies são citadas também por Teston \& Corseuil (2000a) no Centro de Pesquisas e Conservação da Natureza Pró-Mata e por Iserhard \& Romanowski (2004) na região do Vale do rio Maquiné. Hyalenna pascua, característica de matas primárias e secundárias entre 800 e 1700 m de altitude (Willmott \& Lamas 2006), foi encontrada apenas uma vez no interior de uma trilha de mata com reflorestamento de araucária. Possui ocorrência conhecida para florestas de altitude de Minas Gerais, Rio de Janeiro, São Paulo e sul do Paraná (Willmott \& Lamas 2006), e é um registro novo para o Rio Grande do Sul, constituindo-se, assim, em seu limite de distribuição mais ao sul no Brasil. Pseudotinea hemis (Schaus, 1927) (Riodinidae), além de um registro novo para o Rio Grande do Sul, é considerada uma espécie endêmica de ambientes abertos em topos de morro e de florestas de altitude, e ameaçada, principalmente, pela destruição de seu habitat (Hall \& Callaghan 2003), além de potencial indicadora de ambientes preservados. Foi encontrada em maior abundância ao final da primavera e início do verão, junto a arbustos floridos nas bordas de reflorestamentos de araucária. Astraptes erycina (Plötz, 1881) é uma espécie rara, tendo sido registrada duas vezes na FLONA, na borda da mata com reflorestamento de Araucária, sendo encontrada também por Iserhard \& Romanowski (2004).

Actinote alalia (C. Felder \& R. Felder, 1860) pode ser considerado um registro novo para o Estado, a partir do momento em que Francini \& Penz (2006) colocam a identificação de Mabilde (1896) em dúvida. De acordo com Teston et al. (2006), esta citação é somente bibliográfica sem a presença de exemplares em coleções. Actinote alalia foi encontrada em duas trilhas na FLONA, com sete indivíduos, associada a moitas floridas, e exemplares estão depositados nas Coleções de referência de Lepidoptera do Departamento de Zoologia da UFRGS e no Museu de História Natural da Universidade Estadual de Campinas. Pterourus menatius cleotas (Gray, 1832) (Papilionidae) também constitui-se em registro recente para o Rio Grande do Sul, tendo sido citada apenas por Mabilde (1896) e Biezanko (1959a), 
este último autor classificando a espécie como relativamente escassa, associada a clareiras dos matos, sendo freqüente apenas em Santa Catarina e Paraná.

Foram registradas também algumas espécies características de ambientes de campo: Pampasatyrus quies (Berg, 1877), Pampasatyrus periphas (Godart, 1824), Pampasatyrus reticulata (Weymer, 1907) e Pampasatyrus ocelloides (Schaus, 1902), todas pertencentes à Nymphalidae, subfamília Satyrinae. As quatro espécies já haviam sido registradas por Teston \& Corseuil (2002a) no CPCN e Romanowski et al. (2009) registram $P$. ocelloides para a região do Vale do Rio Maquiné, acima de $850 \mathrm{~m}$ de altitude. Pampasatyrus quies e $P$. reticulata parecem estar restritas aos campos de altitude em boas condições de preservação, foram encontradas apenas em locais com elevação superior a $800 \mathrm{~m}$. As borboletas deste gênero podem ser consideradas potenciais indicadoras de campos nativos preservados. Voam baixo e junto ao solo, sendo muitas vezes difíceis de capturar devido à rapidez errática do seu voo (Grazia et al. 2008, Romanowski et al. 2009). Também em ambiente de campo, no presente estudo foi coletado um indivíduo de Brevianta celelata (Hewitson, 1874) (Lycaenidae). Weymer (1894), na primeira compilação de borboletas para o Rio Grande do Sul, registrou um único exemplar de B. celelata. Desde então, esta espécie não havia mais sido registrada para o Estado.

Este trabalho ressalta a importância de Unidades de Conservação e seu entorno como mantenedoras da diversidade biológica, representando refúgios essenciais para o desenvolvimento da fauna local de borboletas. Tendo em vista a crescente substituição das formações vegetais nativas no Estado por monoculturas de exóticas e as lacunas acerca do conhecimento da entomofauna da Floresta com Araucária e dos Campos de Altitude, o presente estudo visa aumentar este conhecimento e torná-lo acessível a pesquisadores e aos administradores desta Floresta Nacional, bem como para outras Unidades de Conservação situadas na região dos Campos de Cima da Serra.

\section{Agradecimentos}

Os autores agradecem aos colegas Ana Kristina Silva, Jessie P. dos Santos, Cristina S. Santiago, Lidiane Fucilini, Daniel S. Castro, Lucas Kaminski, Adriano Cavalleri, Patrick Colombo, Caroline Zank, Luiz Ernesto C. Schmidt, Cristina Rodrigues, Simone Leonardi, Raquel R. Santos, Maria O. Marchiori, Juan Anza, Fernanda Pedone e Francisco Steiner pela amizade e pelo auxílio fundamental ao longo do trabalho. À administração e funcionários da Floresta Nacional de São Francisco de Paula em nome da Sra. Edenice Brandão pela permissão de pesquisa e suporte ao longo das amostragens. Aos doutores André Victor Lucci Freitas, Ronaldo Francini, Olaf Mielke e aos senhores Curtis Callaghan e Alfred Moser pela identificação de exemplares de borboletas. Ao Dr. Olaf Mielke e a Dra. Carla Penz pelos comentários e revisão deste trabalho. Este estudo foi financiado pelos Editais Universais do CNPq n ${ }^{\circ}$ 473838/2006-0 e 472175/2007-6, pela bolsa PQ processo 308292/2007-3 e pela CAPES. As coletas foram realizadas com as licenças do IBAMA nº 070/2006 e n 11990-1. Contribuição ${ }^{\circ} 554$ do Departamento de Zoologia da Universidade Federal do Rio Grande do Sul.

\section{Referências}

BACKES, A. 1999. Condicionamento climático e distribuição geográfica de Araucaria angustifolia (Bertol.) Kuntze no Brasil II. Pesqui. Bot. 49(1):31-52.

BACKES, P. \& IRGANG, B. 2004. Mata Atlântica: as árvores e a paisagem. Editora Paisagem do Sul, Porto Alegre, 396 p.

BEHLING, H. 2002. South and Southeast Brazilian grasslands during Late Quaternary times: a synthesis. Palaeogeogr. Palaeoclimatol. Palaeoecol. 177(1-5):19-27.
BIEZANKO, C.M. 1958. Pieridae da Zona Sueste do Rio Grande do Sul. Arq. Entomol. A 1-15.

BIEZANKO, C.M. 1959a. Papilionidae da Zona Sueste do Rio Grande do Sul. Arq. Entomol. A 1-17.

BIEZANKO, C.M. 1959b. Papilionidae da Zona Missioneira. Arq. Entomol. B 1-12.

BIEZANKO, C.M. 1960a. Pieridae da Zona Missioneira do Rio Grande do Sul. Arq. Entomol. B 1-12.

BIEZANKO, C.M. 1960b. III. Danaidae et Ithomidae da Zona Sueste do Rio Grande do Sul. Arq. Entomol. A 1-6.

BIEZANKO, C.M. 1960c. III. Danaidae et Ithomidae da Zona Missioneira do Rio Grande do Sul. Arq. Entomol. B 1-6.

BIEZANKO, C.M. 1960d. IV. Satyridae, Morphidae et Brassolidae da Zona Sueste do Rio Grande do Sul. Arq. Entomol. A 1-13.

BIEZANKO, C.M. 1960e. IV. Satyridae, Morphidae et Brassolidae da Zona Missioneira do Rio Grande do Sul. Arq. Entomol. B 1-10.

BIEZANKO, C.M. 1963. VI. Hesperiidae da Zona Sueste do Rio Grande do Sul. Arq. Entomol. A 1-25.

BIEZANKO, C.M. \& MIELKE, O.H.H. 1973. Contribuição ao estudo faunístico dos Hesperiidae americanos. IV Espécies do Rio Grande do Sul, Brasil, com notas taxonômicas e descrições de espécies novas (Lepidoptera). Acta Biol. Parana. 2(1-4):51-102.

BIEZANKO, C.M., MIELKE, O.H.H. \& WEDDERHOOF, A. 1978. Contribuição ao estudo faunístico dos Riodinidae do Rio Grande do Sul, Brasil (Lepidoptera). Acta Biol. Parana. 7(1):7-22.

BOGGS, C.L., WATT, W.B. \& EHRLICH, P.R. 2003. Butterflies: ecology and evolution taking flight. The University of Chicago Press, Chicago, $704 \mathrm{p}$.

BOND-BUCKUP, G. 2008. Biodiversidade dos Campos de Cima da Serra. Libretos, Porto Alegre, $146 \mathrm{p}$.

BROWN, K.S. 1996. Diversity of Brazilian Lepidoptera: history of study, methods for measurement, and use as indicator for genetic, specific and system richness. In Biodiversity in Brazil, a first approach (C.E.M Bicudo \& N.A. Menezes, orgs). Instituto de Botânica, CNPq, São Paulo, p. 223-253.

BROWN, K.S. \& FREITAS, A.V.L. 1999. Lepidoptera. In Biodiversidade do Estado de São Paulo, Brasil: invertebrados teerrestres (C.R.F. Brandão \& E.M. Cancello, eds). FAPESP, São Paulo, p. 225-245.

BROWN, K.S. \& FREITAS, A.V.L. 2000a. Diversidade de Lepidoptera em Santa Teresa, Espírito Santo. Bol. Mus. Biol. Mello Leitão 11-12:71-118.

BROWN, K.S. \& FREITAS, A.V.L. 2000b. Atlantic Forest Butterflies: indicators for landscape conservation. Biotropica 32(4b):934-956.

COLWELL, R.K. 2007. Estimates 8.0: statistical estimation of species richness and shared species from samples. University of Connecticut, Connecticut. http://viceroy.eeb.ucon.edu/estimates (último acesso em 15/novembro/2008).

CORSEUIL, E., QUADROS, F.C., TESTON, J.A. \& MOSER, A. 2004. Borboletas (Lepidoptera: Papilionoidea e Hesperioidea) coletadas no Centro de Pesquisa e Conservação da Natureza Pró-Mata. 4: Lycaenidae. Divul. Mus. Ciênc. Tecnol. PUCRS 9(1):65-70.

DESSUY, M.B. \& MORAIS, A.B.B. 2007. Diversidade de borboletas (Lepidoptera: Papilionoidea e Hesperioidea) em fragmentos de Floresta Estacional Decidual em Santa Maria, Rio Grande do Sul, Brasil. Rev. Bras. Zool. 24(1):108-120.

DIMARE, R.A., TESTON, J.A. \& CORSEUIL, E. 2003. Espécies de Adelpha Hübner, 1819 (Lepidoptera, Nymphalidae, Limenitidinae) ocorrentes no Rio Grande do Sul, Brasil. Rev. Bras. Entomol. 47(1):75-79.

FERNANDES, A.V. \& BACKES, A. 1998. Produtividade primária em floresta com Araucaria angustifolia no Rio Grande do Sul. Iheringia, Sér. Zool. 51(1):63-78.

FRANCINI, R.B. \& PENZ, C.M. 2006. An illustrated key to male Actinote from Southeastern Brazil (Lepidoptera, Nymphalidae). Biota Neotrop. 6(1): http://www.Biota neotropica.org.br/v6n1/pt/abstract?identificationkey+bn00606012006 (último acesso em 20/novembro/2008). 
GIOVENARDI, R., DIMARE, R.A., SPONCHIADO, J., ROANI, S.H., JACOMASSA, F.A.F., JUNG, A.B. \& PORN, M.A. 2008. Diversidade de Lepidoptera (Papilionoidea e Hesperioidea) em dois fragmentos de florestas no município de Frederico Westphalen, Rio Grande do Sul, Brasil. Rev. Bras. Entomol. 52(4):599-605.

GRAZIA, J., ROMANOWSKI, H.P., ARAÚJO, P.B., SCHWERTNER, C.F., ISERHARD, C.A., MOURA, L.A. \& FERRO, V.G. 2008. Artrópodos Terrestres. In Biodiversidade dos Campos de Cima da Serra (G. BondBuckup, org.). Libretos, Porto Alegre, p. 76-97.

HALL, J.P.W. \& CALLAGHAN, C.J. 2003. A revision of the new riodinid butterfly genus Pseudotinea (Lepidoptera: Riodinidae). J. Nat. Hist. 37:821-837.

ISERHARD, C.A. \& ROMANOWSKI, H.P. 2004. Lista de espécies de borboletas (Lepidoptera: Papilionoidea \& Hesperioidea) da região do vale do Rio Maquiné, Rio Grande do Sul, Brasil. Rev. Bras. Zool. 21(3):649-662.

KRÜGER, C.P. \& SILVA, E.J.E. 2003. Papilionoidea (Lepidoptera) de Pelotas e seus arredores, Rio Grande do Sul, Brasil. Entomol. Vectores 10(1):31-45.

LAMAS, G. 2004. Atlas of Tropical Lepidoptera: checklist. Association for Tropical Lepidoptera; Scientific Publishers, Gainesville, 439 p. (parte 4A)

LAMAS, G. 2008. La sistemática sobre mariposas (Lepidoptera: Hesperioidea Y Papilionoidea) en el mundo: estado actual y perpectivas futuras. In Contribuiciones taxonómicas en órdenes de insectos hipererdiversos (J.L. Bousquets \& A. Lanteri, orgs). UNAM, Cidade do México, p. 57-70.

LEITE, P.F. 2002. Contribuição ao conhecimento fitoecológico do Sul do Brasil. Ciênc. Ambient. 24(1):51-73.

MABILDE, A.P. 1896. Guia practica para os principiantes collecionadores de insectos, contendo a descripção fiel de perto de mil borboletas com 280 figuras lythographadas em tamanho, formas e desenhos conforme o natural: estudo sobre a vida de insectos do Rio Grande do Sul e sobre a caça, classificação e a conservação de uma collecção, mais ou menos regular. Gundlach Schuldt, Porto Alegre, 238 p.

MARCHIORI, M.O. \& ROMANOWSKI, H.P. 2006. Borboletas (Lepidoptera: Papilionoidea e Hesperioidea) do Parque Estadual do Espinilho e seu entorno, Rio Grande do Sul, Brasil. Rev. Bras. Zool. 23(4):1029-1037.

MIELKE, O.H.H. 1980a. Contribuição ao estudo faunístico dos Hesperiidae americanos. V Nota suplementar: as espécies de Pyrrhopyginae e Pyrginae do Rio Grande do Sul, Brasil (Lepidoptera). Acta Biol. Parana. 8-9:7-17.

MIELKE, O.H.H. 1980b. Contribuição ao estudo faunístico dos Hesperiidae americanos. VI Nota suplementar: as espécies de Hesperiinae do Rio Grande do Sul, Brasil (Lepidoptera). Acta Biol. Parana. 8-9:127-172.

MIELKE, O.H.H. 2005. Catalogue of the American Hesperioidea: Hesperiidae (Lepidoptera). Sociedade Brasileira de Zoologia, Curitiba, 1536 p. (6 vol.)

MORAIS, A.B.B., ROMANOWSKI, H.P., ISERHARD, C.A., MARCHIORI, M.O. \& SEGUI, R. 2007. Mariposas del Sur de Sudamérica (Lepidoptera: Papilionoidea e Hesperioidea). Ciênc. Ambient. 35:29-46.

NEW, T.R. 1997. Are Lepidoptera an effective "umbrella group" for biodiversity conservation? J. Insect Conserv. 1(1):5-12.

OCKINGER, E., ERIKSSON, A.K. \& SMITH, H.G. 2006. Effects of grassland abandonment, restoration and management on butterflies and vascular plants. Biol. Conserv. 133(n):291-300. (dados não existem na publicação)

PAZ, A.L.G., ROMANOWSKI, H.P. \& MORAIS, A.B.B. 2008. Nymphalidae, Papilionidae e Pieridae (Lepidoptera: Papilionoidea) da Serra do Sudeste do Rio Grande do Sul, Brasil. Biota Neotrop. 8(1): http://www. biotaneotropica.org.br/v8n1/pt/abstract?Inventory+bn0160801 2008 (último acesso em 20/novembro/2008).
QUADROS, F.C., DORNELES, A.L. \& CORSEUIL, E. 2004. Ninfalídeos (Lepidoptera, Nymphalidae) ocorrentes no norte da Planície Costeira do Rio Grande do Sul. Biociências 12(2):147-164.

ROMANOWSKI, H.P., ISERHARD, C.A. \& HARTZ, S.M. 2009. Borboletas da floresta com araucária. In Floresta de araucária: ecologia, conservação e desenvolvimento sustentável (C.R. Fonseca, A.F. Souza, A.M. LealZanchet, T. Dutra, A. Backes \& G. Ganade, eds). Holos Editora, Ribeirão Preto, p. 229-240.

SAFFORD, H.D. 1999. Brazilian Páramos I: an introduction to the physical environment and vegetation of the campos de altitude. J. Biogeogr. 26:693-712.

SAFFORD, H.D. 2007. Brazilian páramos IV. Phytogeography of the campos de altitude. J. Biogeogr. 34:1701-1722.

TESTON, J.A. \& CORSEUIL, E. 1998. Lista documentada dos Papilionídeos (Lepidoptera, Papilionidae) do Rio Grande do Sul, Brasil. Biociências 6(2):81-94.

TESTON, J.A. \& CORSEUIL, E. 1999. Borboletas (Lepidoptera, Rhopalocera) ocorrentes no Centro de Pesquisas e Conservação da Natureza Pró-Mata. 1. Papilionidae. Divul. Mus. Ciênc. Tecnol. PUCRS 4:217-228.

TESTON, J.A. \& CORSEUIL, E. 2000a. Borboletas (Lepidoptera, Rhopalocera) ocorrentes no Centro de Pesquisas e Conservação da Natureza Pró-Mata. 2. Pieridae. Divul. Mus. Ciênc. Tecnol. PUCRS 5:143-155.

TESTON, J.A. \& CORSEUIL, E. 2000b. Lista documentada dos Pierídeos (Lepidoptera, Pieridae) do Rio Grande do Sul, Brasil. Biociências 8(2):115-132.

TESTON, J.A. \& CORSEUIL, E. 2001. Ninfalídeos (Lepidoptera, Nymphalidae) ocorrentes no Rio Grande do Sul, Brasil. Biociências 9(1):51-61. (parte I)

TESTON, J.A. \& CORSEUIL, E. 2002a. Borboletas (Lepidoptera, Rhopalocera) ocorrentes no Centro de Pesquisas e Conservação da Natureza Pró-Mata. 3: Nymphalidae. Divul. Mus. Ciênc. Tecnol. PUCRS $7: 1-20$.

TESTON, J.A. \& CORSEUIL, E. 2002b. Ninfalídeos (Lepidoptera, Nymphalidae) ocorrentes no Rio Grande do Sul, Brasil. Biociências 10(1):75-84. (parte II)

TESTON, J.A. \& CORSEUIL, E. 2008a. Ninfalídeos (Lepidoptera, Nymphalidae) ocorrentes no Rio Grande do Sul, Brasil. Biociências 16(1):28-32. (parte IV)

TESTON, J.A. \& CORSEUIL, E. 2008b. Ninfalídeos (Lepidoptera, Nymphalidae) ocorrentes no Rio Grande do Sul, Brasil. Biociências 16(1):33-41. (parte V)

TESTON, J.A. \& CORSEUIL, E. 2008c. Ninfalídeos (Lepidoptera, Nymphalidae) ocorrentes no Rio Grande do Sul, Brasil. Biociências 16(1):42-51. (parte VI)

TESTON, J.A., TOLEDO, K.G. \& CORSEUIL, E. 2006. Ninfalídeos (Lepidoptera, Nymphalidae) ocorrentes no Rio Grande do Sul, Brasil. Biociências 4(2):208-213. (parte III)

WEYMER, G. 1894. Exotische Lepidopteren. VII. Beitrag zur Lepidopterenfauna von Rio Grande do Sul. Stett. Entomol. Zeitung 55(10-12):311-333.

WILLMOTT, K.R. \& LAMAS, G. 2006. A phylogenetic reassessment of Hyalenna Forbes and Dircenna Doubleday, with a revision of Hyalenna (Lepidoptera: Nymphalidae: Ithomiinae). Syst. Entomol. 31:419-468.

Recebido em 03/02/10

Versão reformulada recebida em 15/03/10

Publicado em 24/03/10 